\title{
Light-cone QCD Sum Rules for the $\Lambda$ Baryon Electromagnetic Form Factors and its magnetic moment
}

\author{
Yong-Lu Liu and Ming-Qiu Huang \\ Department of Physics, National University of Defense Technology, Hunan 410073, China
}

(Dated: August 28, 2018)

\begin{abstract}
We present the light-cone QCD sum rules up to twist 6 for the electromagnetic form factors of the $\Lambda$ baryon. To estimate the magnetic moment of the baryon, the magnetic form factor is fitted by the dipole formula. The numerical value of our estimation is $\mu_{\Lambda}=-(0.64 \pm 0.04) \mu_{N}$, which is in accordance with the experimental data and the existing theoretical results. We find that it is twist 4 but not the leading twist distribution amplitudes that dominate the results.

PACS numbers:
\end{abstract}

\section{INTRODUCTION}

Electromagnetic (EM) form factors of the hadron are fundamental objects, for they characterize the internal structure of the composite particle. There were a lot of experimental results on EM form factors of baryons [1, 2, 3, 4] and mesons [5, 6, 7, 8, 9, 10] in the past decades. Due to the complexity of their structure, theoretical investigations on baryons received much less attention than those on mesons, and the existing studies on EM form factors of baryons were mainly focused on the nucleon. In comparison with the nucleon, so far as we know, there are no experimental data for $\Lambda$ at present. Therefore, it is instructive and necessary to study its EM form factors theoretically. Fortunately, there have been experimental result on the magnetic moment and other model-dependent theoretical results on EM form factors to test our estimation indirectly. Van Cauteren et al. have investigated the electric and magnetic form factors of strange baryons in the relativistic constituent-quark model [11]. The chiral perturbation theory [12] and the chiral quark/soliton model [13] have been used to investigate the $\Lambda$ EM form factors at low momentum transfer. The present work is devoted to investigate the $\Lambda$ EM form factors at moderately large momentum transfer and estimate its magnetic moment theoretically.

The matrix element of the EM current between the initial and final $\Lambda$ baryon states can be parameterized in terms of the Dirac and Pauli form factors $F_{1}\left(Q^{2}\right)$ and $F_{2}\left(Q^{2}\right)$ :

$$
\left\langle\Lambda\left(P^{\prime}\right)\left|j_{\mu}^{e m}(0)\right| \Lambda(P)\right\rangle=\bar{\Lambda}\left(P^{\prime}\right)\left[\gamma_{\mu} F_{1}\left(Q^{2}\right)-i \frac{\sigma_{\mu \nu} q^{\nu}}{2 M_{\Lambda}} F_{2}\left(Q^{2}\right)\right] \Lambda(P),
$$

where $j_{\mu}^{e m}=e_{u} \bar{u} \gamma_{\mu} u+e_{d} \bar{d} \gamma_{\mu} d+e_{s} \bar{s} \gamma_{\mu} s$ is the EM current relevant to the baryon. $P$ and $P^{\prime}$ are the four-momenta of the initial and final $\Lambda$ baryon states, $M_{\Lambda}$ is the $\Lambda$ mass, $P^{2}=P^{\prime 2}=M_{\Lambda}^{2}, Q^{2}=-q^{2}=-\left(P-P^{\prime}\right)^{2}$ is the momentum transfer (outgoing photon 
momentum), and $\Lambda(P)$ is the $\Lambda$ spinor. From the experimental point of view, Dirac and Pauli form factors are described equivalently by another set of form factors: electric $G_{E}\left(Q^{2}\right)$ and magnetic $G_{M}\left(Q^{2}\right)$ Sachs form factors:

$$
\begin{aligned}
G_{E}\left(Q^{2}\right) & =F_{1}\left(Q^{2}\right)-\frac{Q^{2}}{4 M^{2}} F_{2}\left(Q^{2}\right), \\
G_{M}\left(Q^{2}\right) & =F_{1}\left(Q^{2}\right)+F_{2}\left(Q^{2}\right) .
\end{aligned}
$$

The normalization of the Sachs form factors at $Q^{2}=0$ is given by the baryon charge $G_{E}(0)=0$ and magnetic moment $G_{M}(0)=\mu_{\Lambda}$. In the Breit frame, $G_{E}\left(Q^{2}\right)$ corresponds to the distribution of the electric charge and $G_{M}\left(Q^{2}\right)$ to the magnetic current distribution.

To give the EM form factors, QCD sum rule method from a three-point correlation function is a useful tool. However, the three-point sum rule has a deficiency which restricts its application and accuracy [14]. Instead of it, the light-cone sum rule (LCSR) method, which has firstly been employed on the nucleon by Braun et al. [15], is utilized to study the $\Lambda$ EM form factors in this paper. LCSR, a hybrid of the traditional sum rule (SVZ sum rule) [16] and the theory of hard exclusive processes, is a useful method to include both hard scattering and soft contributions in QCD. It was developed in late 1980's by making a partial resummation of the operator product expansion (OPE) to all orders [17, 18, 19]. The main difference between SVZ sum rule and LCSR is that the short-distance Wilson OPE in increasing dimension is replaced by the light-cone expansion in terms of distribution amplitudes (see Ref. [20] for a review) of increasing twist. The $\Lambda$ baryon distribution amplitudes, which are the fundamental input parameters in LCSR, have been given in Ref. [21, 22]. In this paper, we give a little correction to the nonperturbative parameter $\lambda_{1}$ which has different sign compared with that in Ref. [22]. To leading order in the QCD coupling $\alpha_{s}$, we give the $Q^{2}$-dependence EM form factors of $\Lambda$ in the range $1 \mathrm{GeV}^{2} \leq Q^{2} \leq 7 \mathrm{GeV}^{2}$ considering contributions up to twist 6 .

Another aim of this manuscript is to estimate the $\Lambda$ magnetic moment. As important intrinsic physical values, the magnetic moments of baryons have been investigated theoretically in the past years by various models [12, 23, 24, 25, 26, 27, 28, 29, 30, 31, 32, 33]. Among these models, QCD sum rule is widely used. The conventional way to calculate the magnetic moments of hadrons in QCD sum rule is to deal with the two-point correlation function in the background of the electromagnetic field at zero momentum transfer. This is the developed QCD sum rule method proposed by Balitsky et al. [34] and Ioffe et al. [35]. In their method, the correlation function is expanded in a constant weak external electromagnetic field $F_{\mu \nu}$, and the sum rule of the term connected with the magnetic moment can be gotten directly. This method has been widely used in calculating the magnetic moments of hadrons [23, 34, 35, 36, 37, 38, 39, 40]. In the present paper, another approach is adopted to evaluate the $\Lambda$ magnetic moment. The basic idea of the approach is to extract the magnetic moment from the calculated EM form factors indirectly. It is assumed that the magnetic form factor divided by the magnetic moment can be described by the dipole 
formula:

$$
\frac{1}{\mu_{\Lambda}} G_{M}\left(Q^{2}\right)=\frac{1}{\left(1+Q^{2} / m_{0}^{2}\right)^{2}}=G_{D}\left(Q^{2}\right) .
$$

Since there are no experimental data available for the form factor, the result is fitted by the above dipole formula, and the two parameters $\mu_{\Lambda}$ and $m_{0}^{2}$ are artificial parameters to be determined simultaneously. After that, we use the fit to estimate the magnetic moment of the baryon. In Ref. [32], the authors have ever calculated the magnetic moment of the $\Lambda$ baryon with LCSR. In their calculations, the magnetic moment can be obtained directly. This comes from the fact that they use the photon distribution amplitudes, which allows for the utilization of LCSR at the point $Q^{2}=0$. However, in our case, the working region of the sum rule cannot extrapolate to the zero point directly. Hence, we have to estimate it with other approaches.

This paper is organized as follows. Section II is devoted to derive the light-cone QCD sum rules of the form factors relevant to the momentum transfer $Q^{2}$, and the necessary distribution amplitudes needed in the calculation are presented in this section. Section III is the numerical analysis part in which the magnetic form factor is fitted by the dipole formula, and the magnetic moment is estimated from the fit. This section also presents the QCD sum rules of the nonperturbative parameters $f_{\Lambda}$ and $\lambda_{1}$. The summary and conclusion are given at the end of this section.

\section{LIGHT-CONE QCD SUM RULES FOR THE EM FORM FACTORS}

\section{A. Leading twist}

The derivation of the sum rules begins with the following correlation function:

$$
T_{\mu}(P, q)=i \int d^{4} x e^{i q \cdot x}\left\langle 0\left|T\left\{j_{\Lambda}(0) j_{\mu}^{e m}(x)\right\}\right| \Lambda(P)\right\rangle,
$$

where the interpolating current of the $\Lambda$ baryon field is chosen to be

$$
j_{\Lambda}(0)=\epsilon_{i j k}\left(u^{i}(0) C \gamma_{5} \not d^{j}(0)\right) \not s^{k}(0) .
$$

The coupling constant is defined by the matrix element of the interpolating current between the vacuum and the $\Lambda$ state:

$$
\left\langle 0\left|j_{\Lambda}\right| \Lambda\right\rangle=f_{\Lambda}(P \cdot z) \not \swarrow \Lambda(P),
$$

where $z$ is a light-cone vector, $z^{2}=0$, and $f_{\Lambda}$ determines the normalization of the leading twist $\Lambda$ distribution amplitude.

In order to get the sum rules, the correlation function (4) needs to be expressed both phenomenologically and theoretically. On one side, by inserting a complete set of interme-

diate states with the same quantum numbers as those of $\Lambda$, and using the definition of the 
form factors in Eq. (11) and the definition in Eq. (66), the hadronic representation of the correlation function is expressed as follows:

$$
\begin{aligned}
z^{\mu} T_{\mu}(P, q)= & \frac{1}{M_{\Lambda}^{2}-P^{2}} f_{\Lambda}\left(P^{\prime} \cdot z\right)\left[2 \left(P^{\prime} \cdot z F_{1}\left(Q^{2}\right)\right.\right. \\
& \left.-\frac{q \cdot z}{2} F_{2}\left(Q^{2}\right)\right) \not \not+\left(P^{\prime} \cdot z F_{2}\left(Q^{2}\right)\right. \\
& \left.\left.+\frac{q \cdot z}{2} F_{2}\left(Q^{2}\right)\right) \frac{\not \not \not q}{M_{\Lambda}}\right] \Lambda(P)+\ldots,
\end{aligned}
$$

where $P^{\prime}=P-q$, and the dots stand for the higher resonance and continuum contributions. Here the contraction of the correlation function by $z^{\mu}$ is adopted to get rid of contributions which are subdominant on the light cone.

On the other side, the correlation function is calculated in perturbation theory at large Euclidean momenta $P^{\prime 2}$ and $q^{2}=-Q^{2}$ in terms of the distribution amplitudes. To the leading order, the theoretical side of the correlation function is

$$
\begin{aligned}
z^{\mu} T_{\mu}(P, q)= & 2(P \cdot z)^{2} \not \subset(P) \int \mathcal{D} \alpha\left\{e_{u} \frac{\mathcal{A}_{1}(\alpha)}{s_{1}-(q-P)^{2}}\right. \\
& \left.+e_{d} \frac{\mathcal{A}_{1}(\alpha)}{s_{2}-(q-P)^{2}}+e_{s} \frac{\mathcal{A}_{1}(\alpha)}{s_{3}-(q-P)^{2}}\right\},
\end{aligned}
$$

where $s_{i}=\left(1-\alpha_{i}\right) P^{2}-\left(1-\alpha_{i}\right) / \alpha_{i} q^{2}+m_{i}^{2} / \alpha_{i}$, and $m_{1,2}=0, m_{3}=m_{s}$. The $\Lambda$ baryon distribution amplitudes are defined by the matrix element of the operator between vacuum and the $\Lambda$ state. The leading order distribution amplitudes are expressed as

$$
\begin{aligned}
& 4\left\langle 0\left|u_{\alpha}^{i}\left(a_{1} x\right) d_{\beta}^{j}\left(a_{2} x\right) s_{\gamma}^{k}\left(a_{3} x\right)\right| \Lambda(P)\right\rangle \\
= & \mathcal{V}_{1}(P C)_{\alpha \beta}\left(\gamma_{5} \Lambda\right)_{\gamma}+\mathcal{A}_{1}\left(P \gamma_{5} C\right)_{\alpha \beta} \Lambda_{\gamma} \\
& +\mathcal{T}_{1}\left(P^{\nu} i \sigma_{\mu \nu} C\right)_{\alpha \beta}\left(\gamma^{\mu} \gamma_{5} \Lambda\right)_{\gamma} .
\end{aligned}
$$

The invariant functions $\mathcal{V}_{1}, \mathcal{A}_{1}$ and $\mathcal{T}_{1}$ can be represented explicitly:

$$
F\left(a_{i} p \cdot x\right)=\int \mathcal{D} \alpha e^{-i p \cdot x \Sigma_{i} \alpha_{i} a_{i}} F\left(\alpha_{i}\right)
$$

The integration measure is defined as follows:

$$
\int \mathcal{D} \alpha=\int_{0}^{1} d \alpha_{1} d \alpha_{2} d \alpha_{3} \delta\left(\alpha_{1}+\alpha_{2}+\alpha_{3}-1\right) .
$$

As the usual procedure in QCD sum rule, by taking into account the dispersion relation and the quark-hadron duality, the hadronic representation of the correlation function is matched with that calculated on the light cone. Then after making the Borel transformation to suppress higher resonance contributions, we get the following light-cone QCD sum 
rules:

$$
\begin{aligned}
f_{\Lambda} F_{1}\left(Q^{2}\right) e^{-\frac{M_{\Lambda}^{2}}{M_{B}^{2}}=} & \left\{e_{u} \int_{\alpha_{1_{0}}}^{1} d \alpha_{1} \int_{0}^{1-\alpha_{1}} d \alpha_{2} \mathcal{A}_{1}\left(\alpha_{1}, \alpha_{2}, 1-\alpha_{1}-\alpha_{2}\right) e^{-\frac{s_{1}}{M_{B}^{2}}}\right. \\
& +e_{d} \int_{\alpha_{2_{0}}}^{1} d \alpha_{2} \int_{0}^{1-\alpha_{2}} d \alpha_{1} \mathcal{A}_{1}\left(\alpha_{1}, \alpha_{2}, 1-\alpha_{1}-\alpha_{2}\right) e^{-\frac{s_{2}}{M_{B}^{2}}} \\
& \left.+e_{s} \int_{\alpha_{3_{0}}}^{1} d \alpha_{3} \int_{0}^{1-\alpha_{3}} d \alpha_{1} \mathcal{A}_{1}\left(\alpha_{1}, 1-\alpha_{1}-\alpha_{3}, \alpha_{3}\right) e^{-\frac{s_{3}}{M_{B}^{2}}}\right\} \\
F_{2}\left(Q^{2}\right)= & 0 .
\end{aligned}
$$

Here $\alpha_{i_{0}}$ connects with the continuum threshold $s_{0}$ :

$$
\alpha_{i_{0}}=\frac{\sqrt{\left(s_{0}+Q^{2}-M_{\Lambda}^{2}\right)^{2}+4\left(Q^{2}+m_{i}^{2}\right) M_{\Lambda}^{2}}}{2 M_{\Lambda}^{2}}-\frac{\left(s_{0}+Q^{2}-M_{\Lambda}^{2}\right)}{2 M_{\Lambda}^{2}}
$$

\section{B. Beyond the leading twist}

It is known that for the nucleon, the leading twist contribution is rather small, while higher twist contributions are dominant [15], which is different from cases of mesons. Therefore, it is necessary to consider contributions of the higher twist distribution amplitudes for the calculation of the $\Lambda$ EM form factors. The usual higher twist contributions come from two different physical origins. First, as the hard quark propagator will receive corrections when considering the background gluon field, there may come contributions from the fourparticle (and five-particle) distribution amplitudes. Second, contributions will arise from the matrix element of the three-quark operator in Eq. (9) if we consider other Lorentz structures on the light cone [22, 41, 42]. As the first source does not play a significant role [15, 43], we only consider the second one in this paper.

In the calculation, only axial-like vector Lorentz structures contribute, so we merely present the following decomposition of the matrix element of the three-quark operator:

$$
\begin{aligned}
& 4\left\langle 0\left|\epsilon^{i j k} u_{\alpha}^{i}\left(a_{1} x\right) d_{\beta}^{j}\left(a_{2} x\right) s_{\gamma}^{k}\left(a_{3} x\right)\right| \Lambda(P)\right\rangle \\
& =\mathcal{A}_{1}\left(P \gamma_{5} C\right)_{\alpha \beta} \Lambda_{\gamma}+\mathcal{A}_{2} M\left(P \gamma_{5} C\right)_{\alpha \beta}(\not x \Lambda)_{\gamma}+\mathcal{A}_{3} M\left(\gamma_{\mu} \gamma_{5} C\right)_{\alpha \beta}\left(\gamma^{\mu} \Lambda\right)_{\gamma} \\
& +\mathcal{A}_{4} M^{2}\left(\not \partial \gamma_{5} C\right)_{\alpha \beta} \Lambda_{\gamma}+\mathcal{A}_{5} M^{2}\left(\gamma_{\mu} \gamma_{5} C\right)_{\alpha \beta}\left(i \sigma^{\mu \nu} x_{\nu} \Lambda\right)_{\gamma}+\mathcal{A}_{6} M^{3}\left(\not \partial \gamma_{5} C\right)_{\alpha \beta}(\not \not \Lambda)_{\gamma} .
\end{aligned}
$$

As the above distributions do not have a definite twist (see Ref. [15, 42] for a review), the invariant functions $\mathcal{A}_{i}$ can be parameterized in terms of distribution amplitudes $A_{1}, \ldots, A_{6}$ 
with a definite twist:

$$
\begin{aligned}
& \mathcal{A}_{1}=A_{1}, \\
& 2 P \cdot x \mathcal{A}_{2}=-A_{1}+A_{2}-A_{3}, \\
& 2 \mathcal{A}_{3}=A_{3}, \\
& 4 P \cdot x \mathcal{A}_{4}=-2 A_{1}-A_{3}-A_{4}+2 A_{5}, \\
& 4 P \cdot x \mathcal{A}_{5}=A_{3}-A_{4}, \\
& (2 P \cdot x)^{2} \mathcal{A}_{6}=A_{1}-A_{2}+A_{3}+A_{4}-A_{5}+A_{6},
\end{aligned}
$$

where $A_{1}$ is twist-3, $A_{2}$ and $A_{3}$ are twist-4, $A_{4}$ and $A_{5}$ are twist-5, and $A_{6}$ is twist-6 distribution amplitude.

These distribution amplitudes are scale dependent and can be expanded into contributions of conformal operators. To the leading order conformal spin accuracy the expansion reads [22, 42]

$$
\begin{aligned}
& A_{1}\left(x_{i}, \mu\right)=-120 x_{1} x_{2} x_{3} \phi_{3}^{0}(\mu), \\
& A_{2}\left(x_{i}, \mu\right)=-24 x_{1} x_{2} \phi_{4}^{0}(\mu), \\
& A_{3}\left(x_{i}, \mu\right)=-12 x_{3}\left(1-x_{3}\right) \psi_{4}^{0}(\mu), \\
& A_{4}\left(x_{i}, \mu\right)=-3\left(1-x_{3}\right) \psi_{5}^{0}(\mu), \\
& A_{5}\left(x_{i}, \mu\right)=-6 x_{3} \phi_{5}^{0}(\mu), \\
& A_{6}\left(x_{i}, \mu\right)=-2 \phi_{6}^{0}(\mu) .
\end{aligned}
$$

With the equation of motion, the six parameters can be expressed in terms of two independent parameters $f_{\Lambda}$ and $\lambda_{1}$. To the leading order conformal spin accuracy, they are expressed as

$$
\begin{aligned}
& \phi_{3}^{0}=\phi_{6}^{0}=-f_{\Lambda}, \\
& \phi_{4}^{0}=\phi_{5}^{0}=-\frac{1}{2}\left(f_{\Lambda}+\lambda_{1}\right), \\
& \psi_{4}^{0}=\psi_{5}^{0}=\frac{1}{2}\left(f_{\Lambda}-\lambda_{1}\right) .
\end{aligned}
$$

Considering the definition of Eq. (14) and Eq. (15), the correlation function (41) is calculated up to twist 6 and described explicitly:

$$
\begin{aligned}
& z^{\mu} T_{\mu}(P, q)=2 e_{u}(P \cdot z)^{2} \not \Lambda(P) \int_{0}^{1} d \alpha_{1}\left\{B_{0}\left(\alpha_{1}\right)+B_{1}\left(\alpha_{1}\right) \frac{M_{\Lambda}^{2}}{\left(s_{1}-(q-P)^{2}\right)}\right. \\
& \left.+B_{2}\left(\alpha_{1}\right) \frac{2 M_{\Lambda}^{4}}{\left(s_{1}-(q-P)^{2}\right)^{2}}\right\} \frac{1}{s_{1}-(q-P)^{2}}+2 e_{u}(P \cdot z)^{2} \not \subset q \Lambda(P) \\
& \times \int_{0}^{1} d \alpha_{1}\left\{E_{1}\left(\alpha_{1}\right)-B_{2}\left(\alpha_{1}\right) \frac{2 M_{\Lambda}^{2}}{\alpha_{1}\left(s_{1}-(q-P)^{2}\right)^{2}}\right\} \frac{M_{\Lambda}}{\alpha_{1}\left(s_{1}-(q-P)^{2}\right)^{2}} \\
& +\left(e_{u} \rightarrow e_{d}, \alpha_{1} \leftrightarrow \alpha_{2}, s_{1} \rightarrow s_{2}, B_{i} \rightarrow C_{i}, E_{1} \rightarrow E_{2}\right) \\
& +\left(e_{u} \rightarrow e_{s}, \alpha_{1} \rightarrow \alpha_{3}, \alpha_{2} \rightarrow \alpha_{1}, s_{1} \rightarrow s_{3}, B_{i} \rightarrow D_{i}, E_{1} \rightarrow E_{3}\right) .
\end{aligned}
$$


Here the following notation is used for convenience:

$$
\begin{aligned}
& B_{0}\left(\alpha_{1}\right)=\int_{0}^{1-\alpha_{1}} d \alpha_{2} A_{1}\left(\alpha_{1}, \alpha_{2}, 1-\alpha_{1}-\alpha_{2}\right), \\
& B_{1}\left(\alpha_{1}\right)=2 \widetilde{A}_{1}\left(\alpha_{1}\right)-\widetilde{A}_{2}\left(\alpha_{1}\right)+\widetilde{A}_{3}\left(\alpha_{1}\right)+\widetilde{A}_{4}\left(\alpha_{1}\right)-\widetilde{A}_{5}\left(\alpha_{1}\right), \\
& B_{2}\left(\alpha_{1}\right)=\widetilde{\widetilde{A}}_{1}\left(\alpha_{1}\right)-\widetilde{\widetilde{A}}_{2}\left(\alpha_{1}\right)+\widetilde{\widetilde{A}}_{3}\left(\alpha_{1}\right)+\widetilde{\widetilde{A}}_{4}\left(\alpha_{1}\right)-\widetilde{\widetilde{A}}_{5}\left(\alpha_{1}\right)+\widetilde{\widetilde{A}}_{6}\left(\alpha_{1}\right), \\
& C_{0}\left(\alpha_{2}\right)=\int_{0}^{1-\alpha_{2}} d \alpha_{1} A_{1}\left(\alpha_{1}, \alpha_{2}, 1-\alpha_{1}-\alpha_{2}\right), \\
& C_{1}\left(\alpha_{2}\right)=2 \widetilde{A}_{1}\left(\alpha_{2}\right)-\widetilde{A}_{2}\left(\alpha_{2}\right)+\widetilde{A}_{3}\left(\alpha_{2}\right)+\widetilde{A}_{4}\left(\alpha_{2}\right)-\widetilde{A}_{5}\left(\alpha_{2}\right), \\
& C_{2}\left(\alpha_{2}\right)=\widetilde{\widetilde{A}}_{1}\left(\alpha_{2}\right)-\widetilde{\widetilde{A}}_{2}\left(\alpha_{2}\right)+\widetilde{\widetilde{A}}_{3}\left(\alpha_{2}\right)+\widetilde{\widetilde{A}}_{4}\left(\alpha_{2}\right)-\widetilde{\widetilde{A}}_{5}\left(\alpha_{2}\right)+\widetilde{\widetilde{A}}_{6}\left(\alpha_{2}\right), \\
& D_{0}\left(\alpha_{3}\right)=\int_{0}^{1-\alpha_{3}} d \alpha_{1} A_{1}\left(\alpha_{1}, 1-\alpha_{1}-\alpha_{3}, \alpha_{3}\right), \\
& D_{1}\left(\alpha_{3}\right)=2 \widetilde{A}_{1}\left(\alpha_{3}\right)-\widetilde{A}_{2}\left(\alpha_{3}\right)+\widetilde{A}_{3}\left(\alpha_{3}\right)+\widetilde{A}_{4}\left(\alpha_{3}\right)-\widetilde{A}_{5}\left(\alpha_{3}\right), \\
& D_{2}\left(\alpha_{3}\right)=\widetilde{\widetilde{A}}_{1}\left(\alpha_{3}\right)-\widetilde{\widetilde{A}}_{2}\left(\alpha_{3}\right)+\widetilde{\widetilde{A}}_{3}\left(\alpha_{3}\right)+\widetilde{\widetilde{A}}_{4}\left(\alpha_{3}\right)-\widetilde{\widetilde{A}}_{5}\left(\alpha_{3}\right)+\widetilde{\widetilde{A}}_{6}\left(\alpha_{3}\right), \\
& E_{1}\left(\alpha_{1}\right)=-\widetilde{A}_{1}\left(\alpha_{1}\right)+\widetilde{A}_{2}\left(\alpha_{1}\right)-\widetilde{A}_{3}\left(\alpha_{1}\right), \\
& E_{2}\left(\alpha_{2}\right)=-\widetilde{A}_{1}\left(\alpha_{2}\right)+\widetilde{A}_{2}\left(\alpha_{2}\right)-\widetilde{A}_{3}\left(\alpha_{2}\right), \\
& E_{3}\left(\alpha_{3}\right)=-\widetilde{A}_{1}\left(\alpha_{3}\right)+\widetilde{A}_{2}\left(\alpha_{3}\right)-\widetilde{A}_{3}\left(\alpha_{3}\right),
\end{aligned}
$$

where the distribution amplitudes with a "tilde" are defined as

$$
\begin{aligned}
& \widetilde{A}\left(\alpha_{1}\right)=\int_{0}^{\alpha_{1}} d \alpha_{1}^{\prime} \int_{0}^{1-\alpha_{1}^{\prime}} d \alpha_{2} A\left(\alpha_{1}^{\prime}, \alpha_{2}, 1-\alpha_{1}^{\prime}-\alpha_{2}\right), \\
& \widetilde{A}\left(\alpha_{2}\right)=\int_{0}^{\alpha_{2}} d \alpha_{2}^{\prime} \int_{0}^{1-\alpha_{2}^{\prime}} d \alpha_{1} A\left(\alpha_{1}, \alpha_{2}^{\prime}, 1-\alpha_{1}-\alpha_{2}^{\prime}\right), \\
& \widetilde{A}\left(\alpha_{3}\right)=\int_{0}^{\alpha_{3}} d \alpha_{3}^{\prime} \int_{0}^{1-\alpha_{3}^{\prime}} d \alpha_{1} A\left(\alpha_{1}, 1-\alpha_{1}-\alpha_{3}^{\prime}, \alpha_{3}^{\prime}\right), \\
& \widetilde{\widetilde{A}}\left(\alpha_{1}\right)=\int_{0}^{\alpha_{1}} d \alpha_{1}^{\prime} \int_{0}^{\alpha_{1}^{\prime}} d \alpha_{1}^{\prime \prime} \int_{0}^{1-\alpha_{1}^{\prime \prime}} d \alpha_{2} A\left(\alpha_{1}^{\prime \prime}, \alpha_{2}, 1-\alpha_{1}^{\prime \prime}-\alpha_{2}\right), \\
& \widetilde{\widetilde{A}}\left(\alpha_{2}\right)=\int_{0}^{\alpha_{2}} d \alpha_{2}^{\prime} \int_{0}^{\alpha_{2}^{\prime}} d \alpha_{2}^{\prime \prime} \int_{0}^{1-\alpha_{2}^{\prime \prime}} d \alpha_{1} A\left(\alpha_{1}, \alpha_{2}^{\prime \prime}, 1-\alpha_{1}-\alpha_{2}^{\prime \prime}\right), \\
& \widetilde{\widetilde{A}}\left(\alpha_{3}\right)=\int_{0}^{\alpha_{3}} d \alpha_{3}^{\prime} \int_{0}^{\alpha_{3}^{\prime}} d \alpha_{3}^{\prime \prime} \int_{0}^{1-\alpha_{3}^{\prime \prime}} d \alpha_{1} A\left(\alpha_{1}, 1-\alpha_{1}-\alpha_{3}^{\prime \prime}, \alpha_{3}^{\prime \prime}\right) .
\end{aligned}
$$

These results stem from the partial integration in $\alpha_{1}, \alpha_{2}$ and $\alpha_{3}$, respectively, which is employed to eliminate the factors $1 /(P \cdot x)^{n}$ in the calculation.

Now by using the Borel transformation and subtraction similarly to that in Ref. [15], 
we arrive at the final sum rules:

$$
\begin{aligned}
f_{\Lambda} F_{1}\left(Q^{2}\right) e^{-\frac{M_{\Lambda}^{2}}{M_{B}^{2}}}= & e_{u}\left\{\int_{\alpha_{1}}^{1} d \alpha_{1}\left\{B_{0}\left(\alpha_{1}\right)+B_{1}\left(\alpha_{1}\right) \frac{M_{\Lambda}^{2}}{M_{B}^{2}}+B_{2}\left(\alpha_{1}\right) \frac{M_{\Lambda}^{4}}{M_{B}^{4}}\right\} e^{-\frac{s_{1}}{M_{B}^{2}}}+\left\{B_{1}\left(\alpha_{1_{0}}\right)\right.\right. \\
& \left.\left.+B_{2}\left(\alpha_{1_{0}}\right) \frac{M_{\Lambda}^{2}}{M_{B}^{2}}-\frac{d}{d \alpha_{1_{0}}} \frac{\alpha_{1_{0}}^{2} M_{\Lambda}^{2} B_{2}\left(\alpha_{1_{0}}\right)}{Q^{2}+\alpha_{1_{0}}^{2} M_{\Lambda}^{2}}\right\} \frac{M_{\Lambda}^{2} \alpha_{1_{0}}^{2}}{Q^{2}+\alpha_{1_{0}}^{2} M_{\Lambda}^{2}} e^{-\frac{s_{0}}{M_{B}^{2}}}\right\} \\
& +e_{d}\left\{\int_{\alpha_{2_{0}}}^{1} d \alpha_{2}\left\{C_{0}\left(\alpha_{2}\right)+C_{1}\left(\alpha_{2}\right) \frac{M_{\Lambda}^{2}}{M_{B}^{2}}+C_{2}\left(\alpha_{2}\right) \frac{M_{\Lambda}^{4}}{M_{B}^{4}}\right\} e^{-\frac{s_{2}}{M_{B}^{2}}}\right. \\
& \left.+\left\{C_{1}\left(\alpha_{2_{0}}\right)+C_{2}\left(\alpha_{2_{0}}\right) \frac{M_{\Lambda}^{2}}{M_{B}^{2}}-\frac{d}{d \alpha_{2_{0}}} \frac{\alpha_{2_{0}}^{2} M_{\Lambda}^{2} C_{2}\left(\alpha_{2_{0}}\right)}{Q^{2}+\alpha_{2_{0}}^{2} M_{\Lambda}^{2}}\right\} \frac{M_{\Lambda}^{2} \alpha_{2_{0}}^{2}}{Q^{2}+\alpha_{2_{0}}^{2} M_{\Lambda}^{2}} e^{-\frac{s_{0}}{M_{B}^{2}}}\right\} \\
& +e_{s}\left\{\int_{\alpha_{3_{0}}}^{1} d \alpha_{3}\left\{D_{0}\left(\alpha_{3}\right)+D_{1}\left(\alpha_{3}\right) \frac{M_{\Lambda}^{2}}{M_{B}^{2}}+D_{2}\left(\alpha_{3}\right) \frac{M_{\Lambda}^{4}}{M_{B}^{4}}\right\} e^{-\frac{s_{3}}{M_{B}^{2}}+\left\{D_{1}\left(\alpha_{3_{0}}\right)\right.}\right. \\
& \left.\left.+D_{2}\left(\alpha_{3_{0}}\right) \frac{M_{\Lambda}^{2}}{M_{B}^{2}}-\frac{d}{d \alpha_{3_{0}}} \frac{\alpha_{3_{0}}^{2} M_{\Lambda}^{2} D_{2}\left(\alpha_{3_{0}}\right)}{Q^{2}+m_{s}^{2}+\alpha_{3_{0}}^{2} M_{\Lambda}^{2}}\right\} \frac{M_{\Lambda}^{2} \alpha_{3_{0}}^{2}}{Q^{2}+m_{s}^{2}+\alpha_{3_{0}}^{2} M_{\Lambda}^{2}} e^{-\frac{s_{0}}{M_{B}^{2}}}\right\},
\end{aligned}
$$

and

$$
\begin{aligned}
& f_{\Lambda} F_{2}\left(Q^{2}\right) e^{-\frac{M_{\Lambda}^{2}}{M_{B}^{2}}}=2\left\{e _ { u } \left\{\int_{\alpha_{1_{0}}}^{1} d \alpha_{1}\left\{\frac{M_{\Lambda}^{2}}{\alpha_{1} M_{B}^{2}} E_{1}\left(\alpha_{1}\right)-\frac{M_{\Lambda}^{4}}{\alpha_{1} M_{B}^{4}} B_{2}\left(\alpha_{1}\right)\right\} e^{-\frac{s_{1}}{M_{B}^{2}}}\right.\right. \\
& +\left\{\frac{1}{\alpha_{1_{0}}} E_{1}\left(\alpha_{1_{0}}\right)-\frac{M_{\Lambda}^{2}}{\alpha_{1_{0}} M_{B}^{2}} B_{2}\left(\alpha_{1_{0}}\right)+\frac{d}{d \alpha_{1_{0}}} \frac{\alpha_{1_{0}} M_{\Lambda}^{2}}{Q^{2}+\alpha_{1_{0}}^{2} M_{\Lambda}^{2}} B_{2}\left(\alpha_{1_{0}}\right)\right\} \\
& \left.\times \frac{\alpha_{1_{0}}^{2} M_{\Lambda}^{2}}{Q^{2}+\alpha_{1_{0}}^{2} M_{\Lambda}^{2}} e^{-\frac{s_{0}}{M_{B}^{2}}}\right\} \\
& +e_{d}\left\{\int_{\alpha_{2_{0}}}^{1} d \alpha_{2}\left\{\frac{M_{\Lambda}^{2}}{\alpha_{2} M_{B}^{2}} E_{2}\left(\alpha_{2}\right)-\frac{M_{\Lambda}^{4}}{\alpha_{2} M_{B}^{4}} C_{2}\left(\alpha_{2}\right)\right\} e^{-\frac{s_{2}}{M_{B}^{2}}}\right. \\
& +\left\{\frac{1}{\alpha_{2_{0}}} E_{2}\left(\alpha_{2_{0}}\right)-\frac{M_{\Lambda}^{2}}{\alpha_{2_{0}} M_{B}^{2}} C_{2}\left(\alpha_{2_{0}}\right)\right. \\
& \left.\left.+\frac{d}{d \alpha_{2_{0}}} \frac{\alpha_{2_{0}} M_{\Lambda}^{2}}{Q^{2}+\alpha_{2_{0}}^{2} M_{\Lambda}^{2}} C_{2}\left(\alpha_{2_{0}}\right)\right\} \frac{\alpha_{2_{0}}^{2} M_{\Lambda}^{2}}{Q^{2}+\alpha_{2_{0}}^{2} M_{\Lambda}^{2}} e^{-\frac{s_{0}}{M_{B}^{2}}}\right\} \\
& +e_{s}\left\{\int_{\alpha_{3_{0}}}^{1} d \alpha_{3}\left\{\frac{M_{\Lambda}^{2}}{\alpha_{3} M_{B}^{2}} E_{3}\left(\alpha_{3}\right)-\frac{M_{\Lambda}^{4}}{\alpha_{3} M_{B}^{4}} D_{2}\left(\alpha_{3}\right)\right\} e^{-\frac{s_{3}}{M_{B}^{2}}}\right. \\
& +\left\{\frac{1}{\alpha_{3_{0}}} E_{3}\left(\alpha_{3_{0}}\right)-\frac{M_{\Lambda}^{2}}{\alpha_{3_{0}} M_{B}^{2}} D_{2}\left(\alpha_{3_{0}}\right)+\frac{d}{d \alpha_{3_{0}}} \frac{\alpha_{3_{0}} M_{\Lambda}^{2}}{Q^{2}+m_{s}^{2}+\alpha_{3_{0}}^{2} M_{\Lambda}^{2}}\right. \\
& \left.\left.\left.\times D_{2}\left(\alpha_{3_{0}}\right)\right\} \frac{\alpha_{3_{0}}^{2} M_{\Lambda}^{2}}{Q^{2}+m_{s}^{2}+\alpha_{3_{0}}^{2} M_{\Lambda}^{2}} e^{-\frac{s_{0}}{M_{B}^{2}}}\right\}\right\} .
\end{aligned}
$$




\section{NUMERICAL ANALYSIS}

\section{A. Determination of the parameters $f_{\Lambda}$ and $\lambda_{1}$}

The parameters $f_{\Lambda}$ and $\lambda_{1}$ can be determined by the QCD sum rule method. The sum rules begin with the following correlation functions:

$$
\Pi\left(q^{2}\right)=i \int d^{4} x e^{i q \cdot x}\left\langle 0\left|T\left\{j_{i}(x) \bar{j}_{i}(0)\right\}\right| 0\right\rangle,
$$

where the interpolating currents are

$$
\begin{aligned}
& j_{1}(x)=\epsilon_{i j k}\left(u^{i}(0) C \gamma_{5} \not d^{j}(0)\right) \not s^{k}(0), \\
& j_{2}(x)=\epsilon_{i j k}\left(u^{i}(x) C \gamma_{5} \gamma_{\mu} d^{j}(x)\right) \gamma_{\mu} s^{k}(x),
\end{aligned}
$$

and following the standard QCD sum rule procedure, the results are given by

$$
\begin{aligned}
(4 \pi)^{4} f_{\Lambda}^{2} e^{-\frac{M_{\Lambda}^{2}}{M_{B}^{2}}}= & \frac{2}{5} \int_{m_{s}^{2}}^{s_{0}} s(1-x)^{5} e^{-\frac{s}{M_{B}^{2}}} d s-\frac{b}{3} \int_{m_{s}^{2}}^{s_{0}} x(1-x)(1-2 x) e^{-\frac{s}{M_{B}^{2}}} \frac{d s}{s}, \\
4(2 \pi)^{4} \lambda_{1}^{2} M_{\Lambda}^{2} e^{-\frac{M_{\Lambda}^{2}}{M_{B}^{2}}}= & \int_{m_{s}^{2}}^{s_{0}} \frac{s^{2}}{2}\left[\left(1-x^{2}\right)\left(1-8 x+x^{2}\right)-12 x^{2} \ln (x)\right] e^{-\frac{s}{M_{B}^{2}}} d s \\
& +\frac{b}{12} \int_{m_{s}^{2}}^{s_{0}}(1-x)^{2} e^{-\frac{s}{M_{B}^{2}}} d s-\frac{4}{3} a^{2} e^{-\frac{m_{s}^{2}}{M_{B}^{2}}}+m_{s} a_{s} \int_{m_{s}^{2}}^{s_{0}} e^{\frac{s}{M_{B}^{2}}} d s,
\end{aligned}
$$

where $x=m_{s}^{2} / s$.

The sum rules reveal that they can only give the absolute values of the two parameters. The relative sign of $f_{\Lambda}$ and $\lambda_{1}$ can be determined by the following correlation function:

$$
\Pi\left(q^{2}\right)=i \int d^{4} x e^{i q \cdot x}\left\langle 0\left|T\left\{j_{1}(x) \bar{j}_{2}(0)\right\}\right| 0\right\rangle
$$

from which the sum rule of $f_{\Lambda} \lambda_{1}^{*}$ is given as follows:

$$
\begin{aligned}
4(2 \pi)^{4} f_{\Lambda} \lambda_{1}^{*} M_{\Lambda} e^{-\frac{M_{\Lambda}^{2}}{M_{B}^{2}}}= & \int_{m_{s}^{2}}^{s_{0}} \frac{m_{s}}{6} s\left[(1-x)\left(3+13 x-5 x^{2}+x^{3}\right)+12 x \ln (x)\right] e^{-\frac{s}{M_{B}^{2}}} d s \\
& +\frac{b}{12} \int_{m_{s}^{2}}^{s_{0}} m_{s}(1-x)\left[1-\frac{1}{3}(1-x)\left(5-\frac{2}{x}\right)\right] e^{-\frac{s}{M_{B}^{2}}} \frac{d s}{s} \\
& +\frac{a_{s}}{24} \int_{m_{s}^{2}}^{s_{0}} e^{-\frac{s}{M_{B}^{2}}} d s .
\end{aligned}
$$

In the numerical analysis we adopt the standard values:

$$
\begin{aligned}
& a=-(2 \pi)^{2}\langle\bar{q} q\rangle=0.55 \mathrm{GeV}^{3} \\
& b=(2 \pi)^{2}\left\langle\alpha_{s} G^{2} / \pi\right\rangle=0.47 \mathrm{GeV}^{4} \\
& a_{s}=m_{0}^{2} a, \quad m_{0}^{2}=0.8 \mathrm{GeV}^{2}
\end{aligned}
$$


The threshold is set to be $s_{0}=1.6^{2} \mathrm{GeV}^{2}$, and the working window for the Borel parameter is $1 \mathrm{GeV}^{2}<M_{B}^{2}<2 \mathrm{GeV}^{2}$. The sum rule (27) shows that $f_{\Lambda} \lambda_{1}^{*}$ is positive. Here $f_{\Lambda}$ is taken to be positive and the parameters have the following numerical values:

$$
\begin{aligned}
& f_{\Lambda}=(5.9 \pm 0.2) \times 10^{-3} \mathrm{GeV}^{2}, \\
& \lambda_{1}=(1.0 \pm 0.3) \times 10^{-2} \mathrm{GeV}^{2} .
\end{aligned}
$$

\section{B. Analysis of the sum rules}

Before the numerical analysis, we firstly specify the input parameters used in the lightcone sum rules. The mass of the $\Lambda$ baryon is given by the Particle Data Group (PDG) [44]: $M_{\Lambda}=1.116 \mathrm{GeV}$. The mass of the strange quark is chosen to be $m_{s}=0.15 \mathrm{GeV}$, and the continuum threshold $s_{0}=2.45-2.65 \mathrm{GeV}^{2}$. For the auxiliary Borel parameter $M_{B}^{2}$, a working window in which the results vary mildly is required. The choice of the Borel parameter should satisfy two conditions simultaneously. On the one hand, as the higher twist contributions are proportional to terms $\left(1 / M_{B}^{2}\right)^{n}(n=1,2, \ldots), M_{B}^{2}$ should be large enough to suppress the higher twist contributions. On the other hand, $M_{B}^{2}$ cannot be too large in case that higher resonance and continuum contributions become dominant. In the calculation, the Borel parameter varies in the range $2 \mathrm{GeV}^{2} \leq M_{B}^{2} \leq 4 \mathrm{GeV}^{2}$. Fig. 1(a) gives the dependence of the magnetic form factors on the Borel parameter at different point of $Q^{2}$. One can see from the figure that the results are almost independent of the Borel parameter.

In the following numerical analysis of the sum rules the Borel parameter is taken to be $M_{B}^{2}=3 \mathrm{GeV}^{2}$, and the nonperturbative parameters $f_{\Lambda}$ and $\lambda_{1}$ are the central values in Eq. (29). Fig. 1(b) gives the dependence of the magnetic form factor $G_{M}\left(Q^{2}\right)$ on the momentum transfer $Q^{2}$. The figure shows that the $Q^{2}$-dependence of the magnetic form factor $G_{M}\left(Q^{2}\right)$ is in accordance with our assumption in Eq. (3). In order to estimate the magnetic moment, the magnetic form factor is fitted by the dipole formula (3). The fits are shown in Fig. 2, from which we evaluate the numerical values of the two parameters simultaneously: $\mu_{\Lambda}=-(0.64 \pm 0.04) \mu_{N}$ and $m_{0}^{2}=(0.89 \pm 0.04) \mathrm{GeV}^{2}$. In Fig. 3(a) we also display the $Q^{2}$-dependence of the value $G_{M} /\left(\mu_{\Lambda} G_{D}\right)$. The parameter $m_{0}^{2}$ used in the dipole formula (3) is the central value obtained above, while the magnetic moment

$\mu_{\Lambda}=0.613 \mu_{N}$ comes from Ref. [44]. It is shown from the figure that the result deviates from 1 when $Q^{2}$ becomes large, which is due to the fact that the absolute value of $G_{M}\left(Q^{2}\right)$ form factor decreases with $Q^{2}$, and the influence of the threshold $s_{0}$ becomes important at large momentum transfer.

Tab. Ilists results of $\Lambda$ magnetic moment from various approaches. In comparison with results in Tab. I, our estimation is in accordance with data provided by PDG and other theoretical predictions.

Fig. 3(b) is the $Q^{2}$-dependence of the electric form factor $G_{E}\left(Q^{2}\right)$. The figure shows 
that the electric form factor changes sign at a finite value of $Q^{2}$, which means that in the large momentum transfer the massive $s$ quark play a more important role in determining the electric density of the baryon. This is different from that of the neutron [15]. A similar

conclusion has been given in Ref. [11]. The difference to our calculation is that the electric form factor is negative at lower $Q^{2}$ in their calculation, while another result from Ref. [13] within the frame-work of the chiral quark/soliton model showed that at small $Q^{2}$ this form factor is positive, which is in contradiction to the result from Ref. [11].

Finally, contributions of distribution amplitudes with different twist are calculated for $G_{M}\left(Q^{2}\right)$, which is shown in Fig. 4. It can be concluded that it is twist 4 but not leading twist contributions that dominate the form factor. This stems from the structure of the leading order distribution amplitude of the $\Lambda$ baryon, which is symmetric on the $d$ and $s$ quark if the mass of $s$ quark approaches zero. Contributions of $d$ and $s$ quark can be canceled by the contribution of $u$ quark in the approach. The results are expected to be better if more information about the distribution amplitudes of the $\Lambda$ baryon is known.

To summarize, we provide a fit approach to predict the magnetic moment of a hadron. The $Q^{2}$-dependence EM form factors of the $\Lambda$ baryon are calculated in the framework of the light-cone sum rule up to twist 6 . The magnetic form factor is fitted by the dipole formula to estimate the magnetic moment of the baryon. Our estimation is in accordance with the existing results. As there lack experimental data on the baryon, we only give theoretical investigations on the form factors as what have been done on nucleons. Analysis on the electric form factor shows that it changes sign at a finite large momentum transfer, which is expected to be tested by the future experiments. Studies on contributions of the distribution amplitudes with different twist show that it is the twist 4 but not the leading twist contributions that dominate the result.

\section{Acknowledgments}

This work was supported in part by the National Natural Science Foundation of China under Contract No.10675167.

[1] R. C. Walker et al., Phys. Rev. D 49, 5671 (1994); L. Andivahis et al., Phys. Rev. D 50, 5491 (1994); M. E. Christy et al. (E94110 Collaboration), Phys. Rev. C 70, 015206 (2004)

[2] J. Arrington, Phys. Rev. C 68, 034325 (2003)

[3] P. E. Bosted et al., Phys. Rev. Lett. 68, 3841 (1992); I. A. Qattan et al., Phys. Rev. Lett. 94, 142301 (2005); P. Bourgeois et al., Phys. Rev. Lett. 97, 212001 (2006)

[4] A. Lung et al., Phys. Rev. Lett. 70, 718 (1993); H. Anklin et al., Phys. Lett. B 428, 248 (1998); G. Kubon et al., Phys. Lett. B 524, 26 (2002) 
[5] C. J. Bebek et al., Phys. Rev. D 9, 1229 (1974); C. J. Bebek et al., Phys. Rev. D 13, 25 (1976); C. J. Bebek et al., Phys. Rev. D 17, 1693 (1978)

[6] E. B. Dally et al., Phys. Rev. Lett. 39, 1176 (1977); W. R. Molzon et al., Phys. Rev. Lett. 41, 1213 (1978) [Erratum-ibid. 41, 1523 (1978 ERRAT, 41,1835.1978)]; E. B. Dally et al., Phys. Rev. Lett. 45, 232 (1980); E. B. Dally et al., Phys. Rev. Lett. 48, 375 (1982)

[7] A. Liesenfeld et al. [A1 Collaboration], Phys. Lett. B 468, 20 (1999)

[8] J. Volmer et al. [The Jefferson Lab F(pi) Collaboration], Phys. Rev. Lett. 86, 1713 (2001)

[9] T. Horn et al. [Fpi2 Collaboration], Phys. Rev. Lett. 97, 192001 (2006)

[10] V. Tadevosyan et al. [Jefferson Lab F(pi) Collaboration], Phys. Rev. C 75, 055205 (2007)

[11] T. Van Cauteren et al., Eur. Phys. J. A 20, 283 (2004); T. Van Cauteren et al., nucl-th/0407017

[12] B. Kubis, T. R. Hemmert and U. G. Meissner, Phys. Lett. B 456, 240 (1999); B. Kubis and U. G. Meissner, Eur. Phys. J. C 18, 747 (2001)

[13] H. C. Kim, A. Blotz, M. V. Polyakov, K. Goeke, Phys. Rev. D 53, 4013 (1996)

[14] V. M. Braun, hep-ph/9801222

[15] V. M. Braun, A. Lenz, N. Mahnke and E. Stein, Phys. Rev. D 65, 074011 (2002); V. M. Braun, A. Lenz, and M. Wittmann, Phys. Rev. D 73, 094019 (2006); A. Lenz, M. Wittmann, and E. Stein, Phys. Lett. B 581, 199 (2004)

[16] M. A. Shifman, A. I. Vainshtein and V. I. Zakharov, Nucl. Phys. B 147, 385 (1979); B 147, 448 (1979); V. A. Novikov, M. A. Shifman, A. I. Vainshtein and V. I. Zakharov, Fortschr. Phys. 32, 11 (1984)

[17] I. I. Balitsky, V. M. Braun and A. V. Kolesnichenko, Nucl. Phy. B 312, 509 (1989); Sov. J. Nucl. Phys. 44, 1028 (1986); ibid. 48, 348, 546 (1988)

[18] V. M. Braun and I. E. Filyanov, Z. Phys. C 44, 157 (1989)

[19] V. L. Chernyak and I. R. Zhitnitskii, Nucl. Phys. B 345, 137 (1990)

[20] P. Colangelo, A. Khodjamirian, CERN-TH/2000-296, BARI-TH/2000-394

[21] V. L. Chernyak, A. A. Ogloblin, and L. R. Zhitnitsky, Z. Phys. C 42, 569 (1989)

[22] M. Q. Huang, D. W. Wang, hep-ph/0608170

[23] J. Pasupathy and J. P. Singh, S. L. Wilson and C. B. Chiu, Phys. Rev. D 36, 1442 (1987)

[24] J. Franklin, Phys. Rev. D 66, 033010 (2002)

[25] B. O. Kerbikov and Y. A. Simonov, Phys. Rev. D 62, 093016 (2000)

[26] S. J. Puglia and M. J. Ramsey-Musolf, Phys. Rev. D 62, 034010 (2000)

[27] N. W. Park and H. Weigel, Nucl. Phys. A 541, 453 (1992)

[28] J. G. Contreras, R. Huerta, L. R. Quintero, Rev. Mex. Fis. 50 (5), 490 (2004)

[29] Y. A. Simonov, J. A. Tjon, J. Weda, Phys. Rev. D 65, 094013 (2002)

[30] J. Bartelski, S. Tatur, Phys. Rev. D 71, 014019 (2005)

[31] T. M. Aliev, I. Kanik and M. Savci, Phys. Rev. D 68, 056002 (2003); T. M. Aliev, A. Ozpineci and M. Savci. Phys. Rev. D 66, 016002 (2002), Erratum-ibid. D 67, (2003) 039901; Phys. 
Rev. D 65, 096004 (2002); Phys. Rev. D 65, 056008 (2002); T. M. Aliev, I. Kanik and M. Savci, Phys. Rev. D 62, 053012 (2000)

[32] T. M. Aliev, A. Ozpineci and M. Savci. Phys. Rev. D 66, 016002 (2002), Erratum-ibid. D 67, (2003) 039901

[33] T. M. Aliev, K. Azizi and A. Ozpineci, arXiv: 0803.4420

[34] I. I. Balitsky and A. V. Yung, Phys. Lett. B129, 328 (1983)

[35] B. L. Ioffe and A. V. Smilga, Nucl. Phys. B 232, 109 (1984)

[36] S. L. Wilson, J. Pasupathy and C. B. Chiu, Phys. Rev. D36, 1451 (1987)

[37] F. X. Lee, Phys. Rev. D 57, 1801 (1998).

[38] S. L. Zhu, W-Y. P. Hwang and Z. S. Yang, Phys. Rev. D 57, 1527 (1998)

[39] A. Samsonov, Phys. Atom. Nucl. 68, 114 (2005) ; Yad. Fiz. 68, 116 (2005)

[40] C. B. Chiu, J. Pasupathy, S. J. Wilson, Phys. Rev. D 33, 1961 (1986)

[41] M. Q. Huang, D. W. Wang, Phys. Rev. D 69, 094003 (2004)

[42] V. Braun, R. J. Fries, N. Mahnke, and E. Stein, Nucl. Phys. B 589, 381 (2000)

[43] M. Diehl, Th. Feldmann, R. Jakob and P. Kroll, Eur. Phys. J. C 8, 409 (1999)

[44] C. Amsler et al. (Particle Data Group), Phys. Lett. B 667, 1 (2008) 


\section{Figure and table captions}

Fig. 1.(a) Dependence of the $\Lambda$ magnetic form factor $G_{M}\left(Q^{2}\right)$ on the Borel parameter. The lines correspond to the points $Q^{2}=1,2,3,5 \mathrm{GeV}^{2}$ from the bottom up with the threshold $s_{0}=2.55 \mathrm{GeV}^{2}$. (b) $Q^{2}$-dependence of the magnetic form factor $G_{M}$. The lines correspond to the threshold $s_{0}=$ $2.45-2.65 \mathrm{GeV}^{2}$ from up down.

Fig. 2. Fittings of the form factor $G_{M}\left(Q^{2}\right)$ by $\mu_{\Lambda} /\left(1+Q^{2} / m_{0}^{2}\right)^{2}$ where the dashed lines are the fits. Figures $(a),(b)$ correspond to threshold $s_{0}=2.45,2.65 \mathrm{GeV}^{2}$, respectively.

Fig. 3. The $Q^{2}$-dependence of the form factor $G_{M} /\left(\mu_{\Lambda} G_{D}\right)$ (a) and $G_{E}$ (b). The lines correspond the threshold $s_{0}=2.45,2.55,2.65 \mathrm{GeV}^{2}$ from up down.

Fig. 4. Contributions of different twist for $G_{M}\left(Q^{2}\right)$ at the threshold $s_{0}=$ $2.55 \mathrm{GeV}^{2}$. The dotted line, dashed line and the solid line correspond to twist 3 , twist 4 and all contributions, respectively.

Tab. [1. The magnetic moment of the $\Lambda$ baryon from various models. 


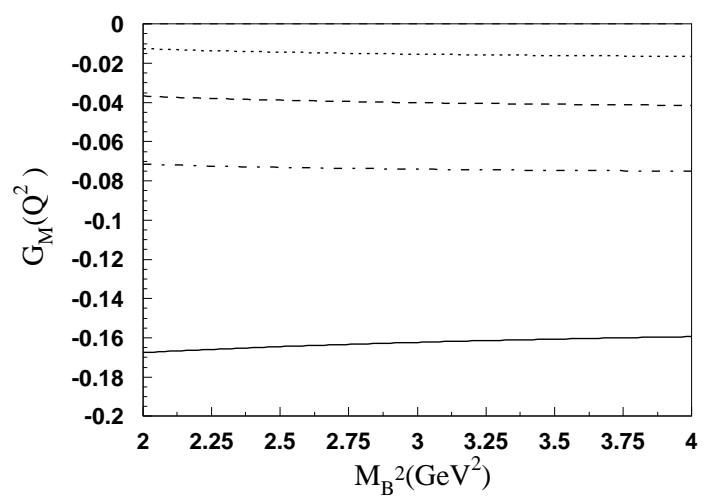

(a)

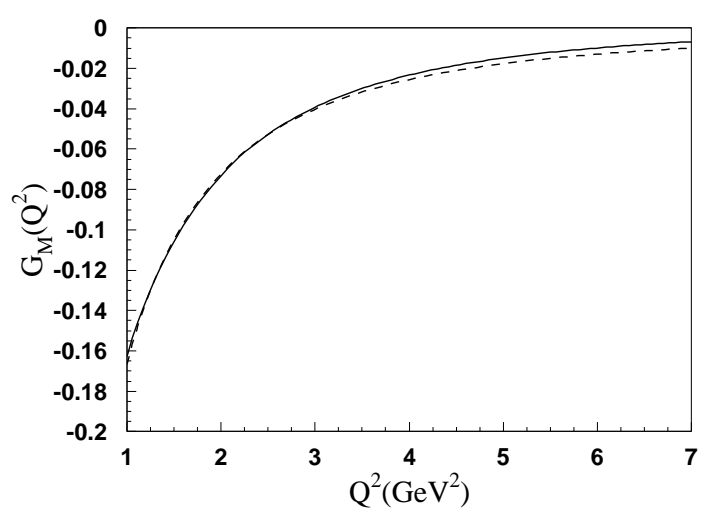

(a)

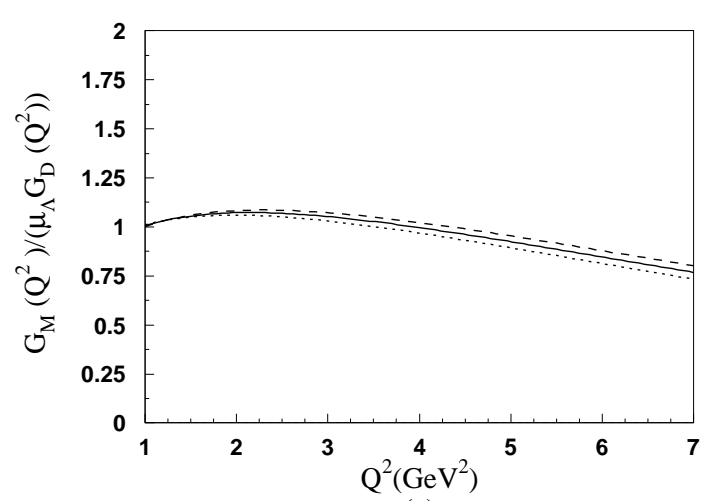

(a)

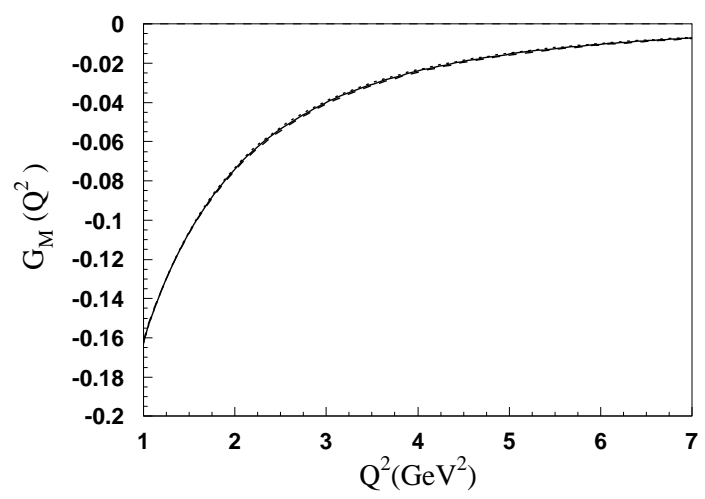

(b)

FIG. 1:

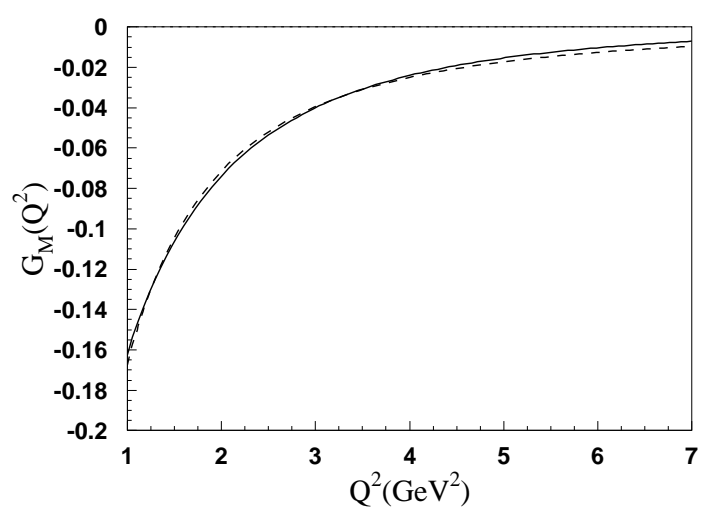

(b)

FIG. 2:

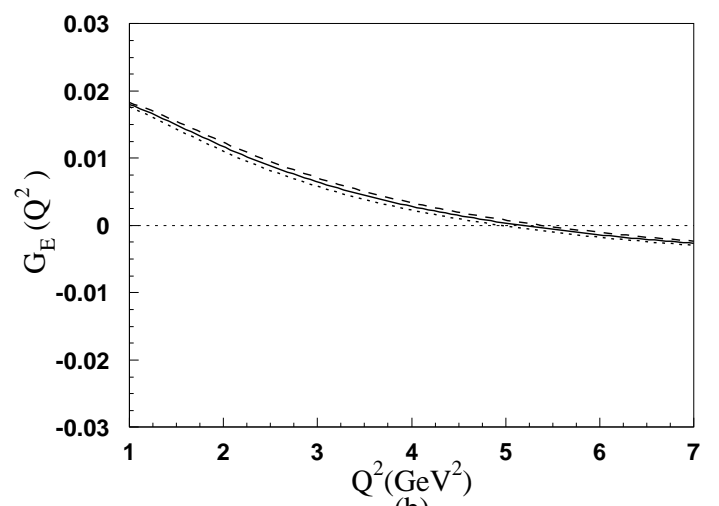

(b)

FIG. 3: 


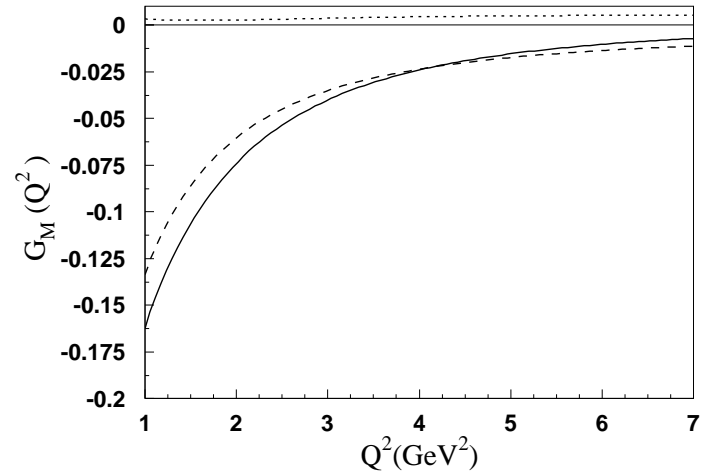

FIG. 4: 
TABLE I:

\begin{tabular}{|c|c|c|c|c|c|c|c|c|c|c|}
\hline Model & $\begin{array}{c}\text { PDG } \\
{[44]}\end{array}$ & $\begin{array}{c}\text { QCDSR } \\
{[23]}\end{array}$ & $\begin{array}{c}\text { SQM } \\
{[24]}\end{array}$ & $\begin{array}{c}\text { QCDSA } \\
{[25]}\end{array}$ & $\begin{array}{c}\chi \text { PT } \\
{[26]}\end{array}$ & $\begin{array}{c}\text { SKRM } \\
{[27]}\end{array}$ & $\begin{array}{c}\text { NQM } \\
{[28]}\end{array}$ & $\begin{array}{c}\text { EQLA } \\
{[29]}\end{array}$ & $\begin{array}{c}\text { GSE } \\
{[30]}\end{array}$ & $\begin{array}{c}\text { LCSR } \\
{[31]}\end{array}$ \\
\hline$\mu_{\Lambda}\left(\mu_{N}\right)$ & -0.613 & $-0.50 /$ & -0.67 & -0.69 & -0.613 & -0.60 & -0.63 & -0.60 & -0.606 & -0.7 \\
& & -0.54 & & & & & & & & \\
\hline
\end{tabular}

